\title{
Post Penetrating Keratoplasty Glaucoma: An Overview
}

\author{
Anuradha $\mathbf{R}^{*}$ \\ HIHT University, India
}

*Corresponding author: Anuradha Raj, HIHT University, India, Email:

dranuradha_sagar@yahoo.com

\section{Research Article}

Volume 4 Issue 2

Received Date: May 20, 2019

Published Date: June 19, 2019

DOI: 10.23880 /oajo-16000183

\section{Abstract}

Glaucoma is the leading cause of blindness after penetrating keratoplasty (PK) and its management is still controversial. Earlier diagnosis is mandatory to salvage the graft. The newer modalities like recent tonopens, ultrasound biomicroscopy (UBM), Anterior segment optic coherence tomography (ASOCT) are helpful tools for its diagnosis. Recent developments in its management includes newer drugs, surgical procedures such as trabeculectomy with mitomycin-C, glaucoma drainage devices (GDD), and cyclodestructive procedures with Nd: YAG (neodymium: yttrium-aluminium-garnet) and diode lasers. Despite all these advances the risk of graft failure continues to be high.

Keywords: Post-Keratoplasty Glaucoma; Anterior Segment Optic Coherence Tomography; Trabeculectomy with Mitomycin C

Abbreviations: PK: Penetrating Keratoplasty; UBM: Ultrasound Biomicroscopy; ASOCT: Anterior Segment Optic Coherence Tomography; GDD: Glaucoma Drainage Devices; PAS: Peripheral Anterior Synechia; DCT: Dynamic Contour Tonometer; GAT: Goldmann Applanation Tonometre; CCT: Central Corneal Thickness; ACD: Anterior-Chamber Depth; ALT: Argon Laser Trabeculoplasty.

\section{Introduction}

Allograft rejection and severe astigmatism are well known complications of PK but post keratoplasty glaucoma (PKG) is one of the most common cause for irreversible visual loss [1] and the second leading cause for graft failure due to significant endothelial cell loss especially in patients who have already low endothelial reserve. The amount of cell loss appears to correlate with the duration of the increased IOP. Early diagnosis of PKG is mandatory to preserve optimal graft clarity and optic nerve head function [2]. PKG is defined as an elevated IOP greater than $21 \mathrm{mmHg}$ within one month of PK with or without associated visual field loss or optic nerve head changes. Tonometry is difficult in these cases because of thick or astigmatic corneal graft. Assessment of the optic nerve and visual field before or after surgery is nearly impossible because of preoperative hazy media and postoperative high corneal astigmatism which makes the diagnosis difficult [3].

\section{Incidence}

In 1969, Irvine and Kaufman were first to report the high incidence of increased IOP following PK with a mean pressure of $40 \mathrm{mmHg}$ in aphakic transplants and 50 mmHg in combined transplants and cataract extraction in the immediate post-operative period [4]. Goldberg reported about $30 \%$ incidence of increased IOP in postoperative period in patients with aphakic bullous keratopathy. Regrafts also showed high incidence of raised IOP both in the early (45\%) and late post-operative phase $(52 \%)$ and those with pre-existing glaucoma $(71 \%)$ [5]. Kirkness reported a higher incidence of glaucoma in patients undergoing PK following corneal perforation, suppurative keratitis as a result of peripheral anterior synechia (PAS) formation and secondary angle closure. 
The incidence of PKG differs significantly from $0-12 \%$ to $75 \%$ respectively [6].

\begin{tabular}{|c|c|}
\hline \multicolumn{2}{|c|}{ Risk factors for PKG } \\
\hline 1. & Aphakic and pseudophakic bullous keratopathy \\
\hline 2. & Mesodermal dysgenesis \\
\hline 3. & Irido-corneal-endothelial syndrome \\
\hline 4. & Pre-existing glaucoma \\
\hline 5. & Perforated corneal ulcer \\
\hline 6. & Adherent leukoma \\
\hline 7. & Regraft \\
\hline 8. & Post-traumatic cases \\
\hline 9. & Combined PK and cataract extraction \\
\hline 10. & Vitrectomy during PK \\
\hline
\end{tabular}

\begin{tabular}{|r|c|}
\hline \multicolumn{2}{|c|}{$\begin{array}{c}\text { Operative factors responsible for raised IOP } \\
\text { postoperatively }\end{array}$} \\
\hline 1. & Tight and superficial suturing with long bites \\
\hline 2. & Larger recipient bed with same size donor button \\
\hline 3. & Increased peripheral corneal thickness \\
\hline 4. & Retained viscoelastic \\
\hline
\end{tabular}

\begin{tabular}{|c|c|}
\hline \multicolumn{2}{|c|}{$\begin{array}{c}\text { Causes of elevated IOP after PK in early postoperative } \\
\text { period }\end{array}$} \\
\hline 1. & Post-operative inflammation \\
\hline 2. & Viscoelastic substances \\
\hline 3. & Wound leakage \\
\hline 4. & Hyphema \\
\hline 5. & Pupillary-block glaucoma \\
\hline 6. & Preexisting glaucoma \\
\hline 7. & PK in aphakic eyes \\
\hline 8. & PK combined with cataract extraction \\
\hline \multicolumn{2}{|c|}{$\begin{array}{c}\text { Causes for elevated IOP in the late postoperative } \\
\text { period }\end{array}$} \\
\hline 1. & PK in aphakic eyes \\
\hline 2. & PK combined with cataract extraction \\
\hline 3. & Chronic angle-closure glaucoma \\
\hline 4. & Pre-existing glaucoma \\
\hline 5. & Steroid induced glaucoma \\
\hline 6. & Graft rejection with glaucoma \\
\hline 7. & Ghost cell glaucoma \\
\hline 8. & Malignant glaucoma \\
\hline
\end{tabular}

\section{Pathogenesis}

PKG may develop as a result of number of mechanical factors, sequelae of inflammatory conditions leading to PAS (Figure 1A \& 1B) wound closure technique and postoperative pharmacological agents. Olson and Kaufman proposed that the elevated IOP following PK in an aphakic patient might be the result of angle distortion due to compression of the tissue in the angle which compromise the trabecular meshwork function, and the situation is further aggravated by tight suturing, long bites (more compressed tissue), larger trephine sizes, smaller recipient corneal diameter and increased peripheral corneal thickness [7]. Zimmerman postulated that the trabeculum needs posterior fixation by the ciliary body-lens support system and an anterior support by the Descemet's membrane. In aphakia, the posterior support is relaxed with the removal of the lens and anterior support is lost after incision of descemet's membrane after PK. Both these factors lead to a partial trabecular collapse and obstruction of aqueous outflow leading to PKG [8]. In view of this theory, Zimmerman reported that oversized donor buttons $(0.5-1 \mathrm{~mm}$ larger than the host bed) in aphakic patients reduced the incidence of glaucoma. The effect was more obvious when an 8-mm donor button was used in a 7.5-mm host bed [9]. Retained viscoelastic material also increase IOP in the early postoperative period by clogging the trabecular meshwork.

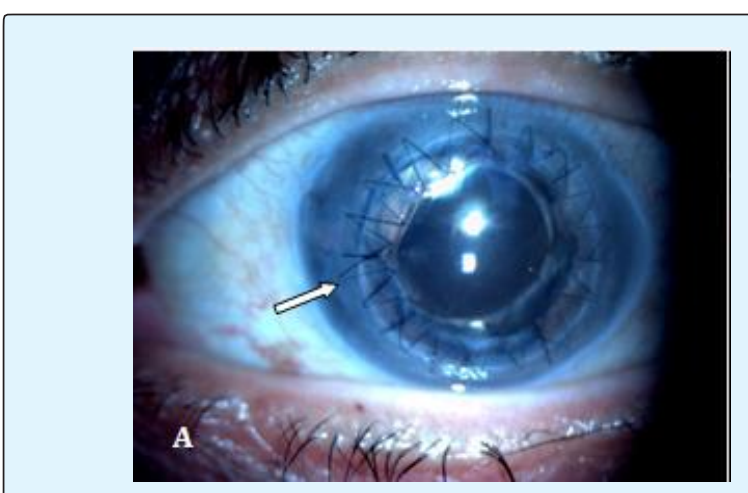

Figure 1A: Iridocorneal adhesions on temporal side of the graft.

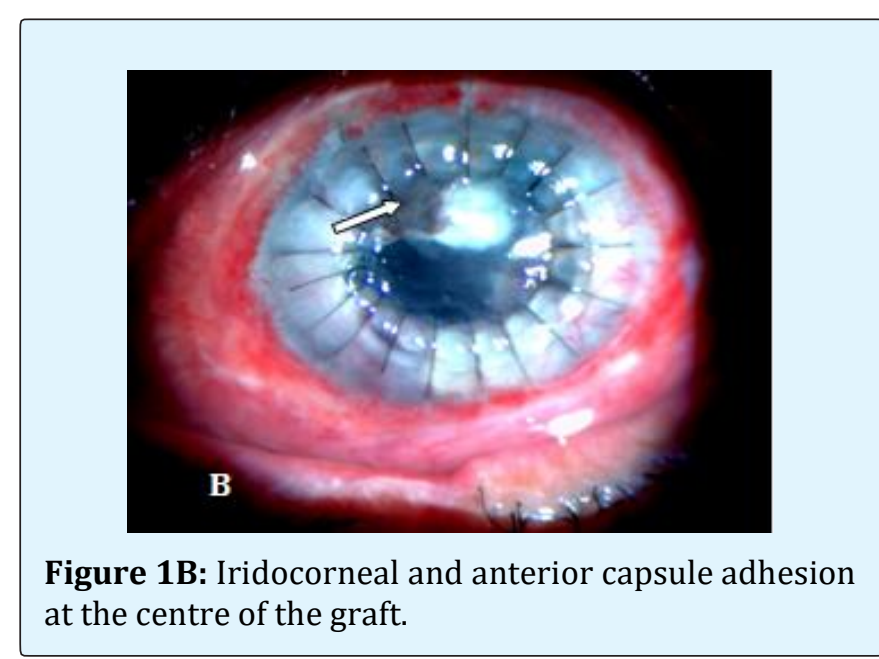




\section{Diagnosis}

Changes in corneal thickness, post-operative astigmatism and refractive changes after PK often preclude reliable post-operative assessment of IOP, disc and visual fields. Stereoscopic optic nerve head evaluation under mydriasis is advocated. Gonioscopy may be performed to view the site and extent of PAS. In early post-operative period, when the corneal surface is irregular, IOP can be measured with the Mackay-Marg electronic applanation tonometer, the pneumatic applanation tonometer, tono-pen, or recent dynamic contour tonometer (DCT) independent of the corneal thickness [10]. Goldmann applanation tonometre (GAT) can be used if graft surface is smooth with intact epithelium. Rotation of the prism is recommended so that the red mark on the prism holder is set at the flatter meridian of the cornea (along the negative axis) to obtain accurate reading with GAT. Alternately two pressure readings taken 90 degrees apart can be averaged. The accuracy of GAT is reduced in corneal edema, scars, blood staining or any condition that thickens or alters the corneal elasticity [11]. Corneal edema predispose to false low readings and corneal scar will record false high. IOP measurement with GAT is standardized for a central corneal thickness (CCT) of $520 \mu$, overestimation of IOP may occur due to an increase in the corneal thickness. In cases with complete tarsorrhaphy, the IOP can be estimated by digital tonometry [12] though preview phosphene tonometer is also available to measure IOP through the lid structures.

UBM is useful to assess the angle and anteriorsegment anatomy in opaque corneas where the details are not clearly visible. It provides an accurate objective quantification of angles, anterior-chamber depth (ACD) assessment, location and extent of iridocorneal adhesions (Figures 2a \& 2b) phakic/aphakic status, IOL position, and corneal graft thickness. UBM helps to diagnose relative pupillary block, PAS, plateau iris configuration.

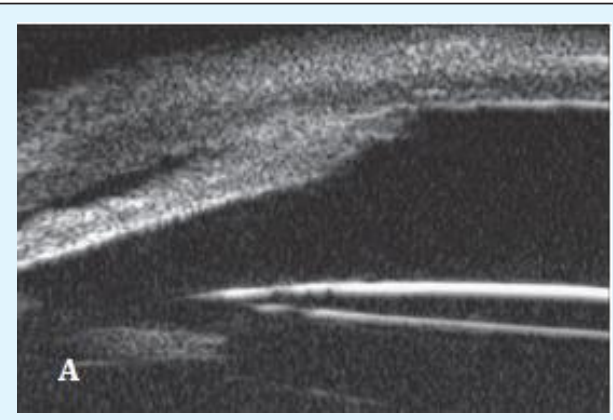

Figure 2A: UBM showing graft host junction synechiae.

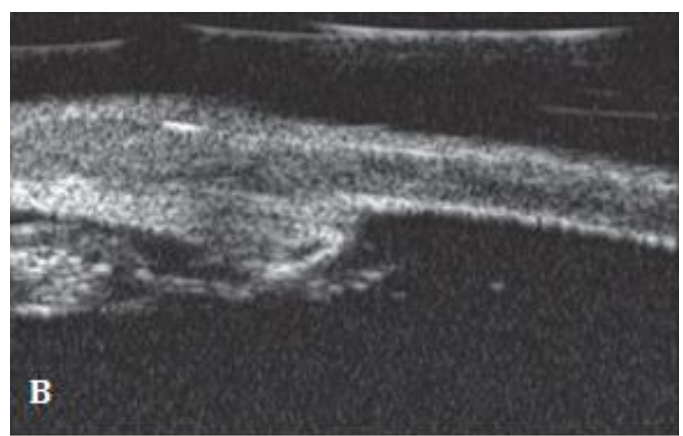

Figure 2B: UBM showing peripheral anterior synechiae.

It helps to decide the site of trabeculectomy and plan the quadrant of placement of the tube of the GDD in the AC because of poor visibility which can help to enhance the success rate of the filtering/drainage implant procedure before repeat grafting is done to restore vision. Secondary angle closure caused by PAS formation is one of the important cause for PKG in eyes with opaque grafts [13].

ASOCT provides non-contact, non-invasive, highresolution, real-time cross-sectional images of the anterior segment of the eye. It enables detailed visualization of the anterior segment and angle anatomy in eyes with opaque corneal grafts and secondary glaucoma. The longer wavelength light scatters less in opaque tissues, allows deeper penetration and permits imaging through the limbus to visualise angle structures such as the scleral spur and angle recess. The commonly used quantitative parameters are as angle opening distance, angle recess, trabecular iris space area, iris thickness, AC width [14] (Figure 3).

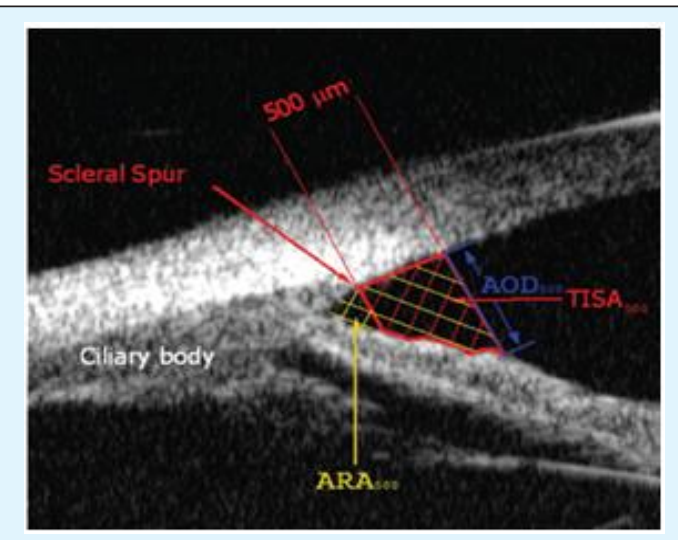

Figure 3: Angle opening distance (AOD), angle recess area (ARA), trabecular iris space area (TISA). 
Pre-operatively exact site of PAS may be helpful in deciding the site of trabeculectomy or placement of glucoma drainage devices (GDD). ASOCT helps in the immediate post-operative follow up of these patients and provides rapid and high-resolution images of the entire circumference of the AC angle, PAS (Figure 4a,4b \& 4c), posterior synechiae (with IOL) and obstruction of an $\mathrm{AC}$ drainage tube [15]. Angle evaluation using ASOCT is recommended in all eyes with PKG before planning any corneal or glaucoma intervention [16].

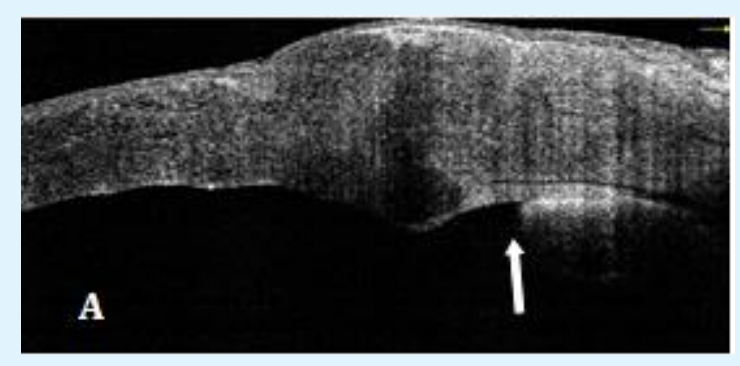

Figure 4A: ASOCT arrow showing peripheral anterior synechiae near graft host junction.

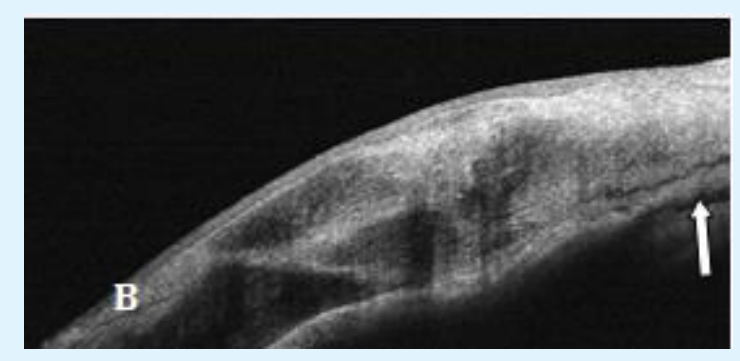

Figure 4B: ASOCT arrow showing anterior synechiae involving central part of the graft near pupil.

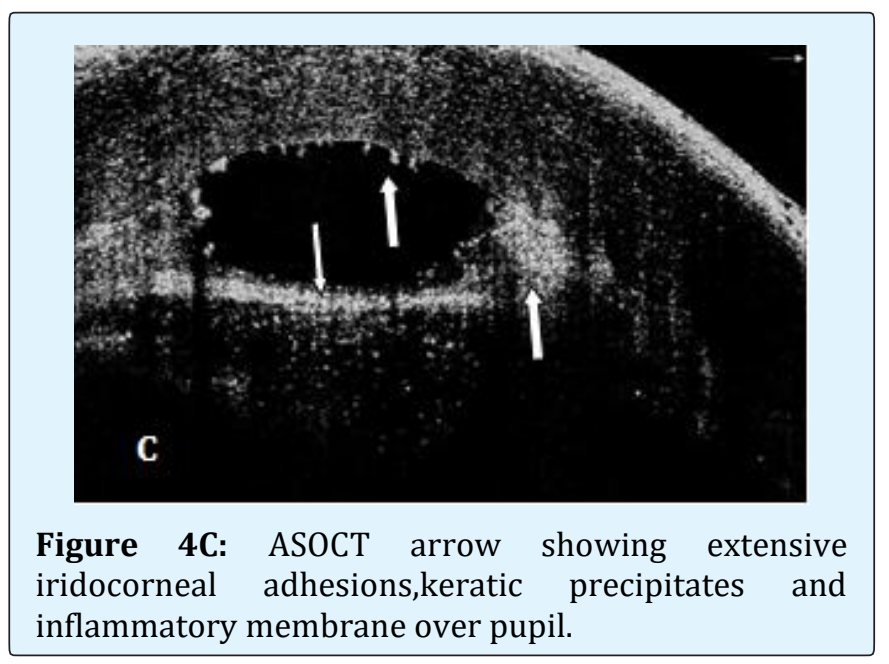

Anuradha R. Post Penetrating Keratoplasty Glaucoma: An Overview. J Ophthalmol 2019, 4(2): 000183.
Management: Markedly elevated IOP in the postoperative period could compromise the graft, both by causing endothelial cell damage and decreasing the corneal sensations secondary to angle closure glaucoma. Therefore, it should be prevented and treated aggressively.

\section{Preventive Measures}

Pre-existing glaucoma is a risk factor for graft failure in aphakic and pseudophakic eyes and is difficult to treat [17]. Reinhard reported the 3-year graft survival rate in $71 \%$ patients with a pre-existing glaucoma in contrast to $89 \%$ without such history [18]. Use of an oversized donor button $(0.5-1 \mathrm{~mm})$ provides good AC depth and lower incidence of PAS which decreases chances of postoperative glaucoma [19]. Deep suture bites increases the aqueous outflow. Goniosynechiolysis, iridoplasty (iris tightening procedure) in cases of a floppy iris, removal of viscoelastic material at the end of the surgery and careful wound closure are the factors which prevent PKG. Postoperatively judicious use of steroids controls the inflammation and prevents PAS. Mobility of pupil should be ensured to prevent pupillary block glaucoma.

\section{Medical Management of Established PKG}

Topical medication is still the first line of treatment in cases of PKG. Beta-adrenergic blocking agents (timolol, betaxolol) act by decreasing the aqueous production. Their side effects include superficial punctate keratopathy, corneal anesthesia, damage to the ocular surface and dry eyes by impairing the quantity and quality of the mucus layer of tear film which adversely affect the graft epithelium and compromise graft function. Their cautious use in aphakic and pseudophakic patients is advocated as they can produce cystoid macular edema. Brimonidine tartrate $0.2 \%$ a relatively selective alpha- 2 adrenergic agonist is better tolerated than apraclonidine hydrochloride and is useful in controlling IOP. Apraclonidine $0.5 \%$ is a potent anterior segment vasoconstrictor and is useful both to prevent $\mathrm{AC}$ bleeding during surgery and to control the pressure spike resulting from such a bleed so one drop of apraclonidine $0.5 \%$ is recommended 1 hour before surgery and 12 hours postoperatively. Allergic reactions are known with it for long term use. Miotics can induce uveitis which may initiate graft rejection and increase the risk of a retinal detachment hence not recommended for aphakic eyes. Topical carbonic anhydrase inhibitors like dorzolamide and brinzolamide lead to irreversible corneal decompensation as they suppress the carbonic anhydrase enzyme in corneal endothelium so should not be used in 


\section{Open Access Journal of Ophthalmology}

patients with a past history of graft rejection or with limited endothelial cell counts [20]. Systemic carbonic anhydrase inhibitors are useful in the treatment of pressure spikes in the immediate postoperative period but should be used cautiously in elderly patients because of their side effects such as paresthesias, tinnitus, gastrointestinal disturbances, fatigue, depression, anorexia, and weight loss [21].

Prostaglandin analogs decrease IOP by increasing the uveoscleral outflow and can be used with beta-blockers and carbonic anhydrase inhibitors but should not be used in patients with history of herpes simplex keratitis, uveitis, and aphakia and pseudophakia as it can cause cystoid macular edema. Benzalkonium chloride (BAC $0.01 \%$ concentration) is the preservative used in the majority of these topical medications and can have toxic effects on the corneal epithelium by damaging cell wall and microvilli leading to its increased permeability. In the case of a steroid-responsive glaucoma, the dose of steroid drops may be tapered to the minimum required. Alternatively stronger steroid drops such as prednisolone acetate can be replaced by steroids with less tendency to increase IOP, (e.g., topical fluorometholone, loteprednol etabonate $0.5 \%$ or $0.2 \%$ ). Topical cyclosporin A $0.5 \%$ may also help to control the pressure. Perry etal have reported a mean reduction of IOP by $8.7 \mathrm{~mm} \mathrm{Hg}$ after topical corticosteroids were replaced by topical cyclosporin A $0.5 \%$ in $21(84 \%)$ of their 25 patients. Graft clarity was maintained in all patients, with one allograft rejection episode that responded to hourly cyclosporin drops [22]. Preferably preservative free drugs should be used for longer term to protect the corneal epithelium.

\section{Surgical Management}

- Laser trabeculoplasty

- Trabeculectomy with antimetabolites

- GDDs

- Cyclodestructive procedures

\section{Laser Trabeculoplasty}

Argon laser trabeculoplasty (ALT) is usually employed in patients with open angle glaucoma, clear grafts with moderately elevated IOP $(20-25 \mathrm{mmHg})$ and in patients whose IOP is not adequately controlled with topical medications. Van meter reported its successful use to treat PKG in aphakic and pseudophakic eyes [23]. ALT has its limitation in presence of PAS and poor visibility of the trabecular meshwork. ALT and selective ALT have limited data to support their efficacy in PKG.

\section{Trabeculectomy}

Conventional trabeculectomy is usually not effective in PKG due to dense perilimbal scarring, extensive PAS and shallow AC. The failure rate is further increased in aphakic eyes due to blockage of the trabeculectomy ostium by the vitreous. The anti-metabolites (5fluorouracil [5FU] and mitomycin-C [MMC]) improves IOP control and the success of trabeculectomy by its antifibroblastic activities. Five milligrams of $5 \mathrm{FU}$ in $0.1 \mathrm{cc}$ is given daily as a subconjunctival injection in the immediate post-operative period for 7-10 days. Corneal epithelial toxicity needs special care for usage of these antimetabolites [24].

Mitomycin-C $0.04 \%$ can be applied for $2-4 \mathrm{~min}$ subconjunctivally or sub-sclerally which increases the success rate of filtering surgery. Misaki etal showed $73.0 \%$ success after trabeculectomy with mitomycin C without glaucoma medications. Most of the trabeculectomy patients without mitomycin C failed within 6 months. Persistent epithelial defect, cystoid macular oedema, choroidal detachment, leaking bleb and graft rejection had been reported [25]. Mitomycin-C should be thoroughly washed off prior to entry into the AC. Ayyala reported $77 \%$ success rate in IOP control with use of mitomycin-C in trabeculectomy patients of PKG [26].

Raj et al reported good IOP control in trabeculectomy with releasable suture till three months as compared to trabeculectomy augmented by MMC but comparable resultsat six months of follow up [27].

\section{Glaucoma Drainage Devices}

GDD create an alternate pathway by channeling aqueous from the $\mathrm{AC}$ through a long tube to an equatorial plate that promotes bleb formation. Kirkness in 1987 was the first to report the use of GDD in PKG. These devices had good control of glaucoma but the risk of graft rejection was high [28]. Kirkness hypothesized that GDD allow retrograde passage of the inflammatory cells into the AC. Post-surgical uveitis, extensive PAS, and multiple previous surgeries may be the other factors to compromise the graft [29]. Sherwood reported good IOP control with GDD after two years of follow up i.e upto $96 \%$ [30]. The optimal location for the drainage tube remains controversial but the its most common location is in the AC. Arroyave found no difference in IOP control between the AC and pars plana tube placement, but $83 \%$ of grafts remained clear with tubes in pars plana as compared to $48 \%$ into AC. 
Tube into the vitreous cavity significantly reduces its interaction with graft which leads to improvement in graft survival [31]. Sidoti studied pars plana tube insertion after pars plana vitrectomy and found IOP control in $62 \%$ and clear grafts in $41 \%$ of the eyes after 2 years but was associated with higher rate of posterior segment complications [32]. Ritterband studied 83 eyes in which the drainage tube was either placed in the pars plana primarily or moved from the AC to the pars plana at the time of PK and reported IOP control in 83\% and graft clarity in $59 \%$ of patients after 2 years [33]. Shunt tubes can be occluded in both places so placement of the tube into the vitreous cavity necessitates a complete vitrectomy with removal of the vitreous skirt which prevents vitreous from occluding the tube. Well-placed tube in the AC away from the cornea allows its observation at the slit lamp and easy access for laser and needling to remove any tissues if the tube tip is blocked

\section{Cyclodestructive Procedures}

It is employed to lower IOP by decreasing aqueous humour production by ablating the portion of ciliary body. Destruction of the ciliary body can be achieved through transscleral application of cryotherapy or transscleral or endoscopic delivery of diode, krypton or $\mathrm{Nd}$ : YAG laser. The primary aim of this therapy is to lower IOP and to preserve visual function, graft clarity, as well as diminish the number of antiglaucomatous medications required. Recent studies showed success rate of $72 \%$ after a year of single treatment of diode laser in terms of improvement in visual acuity, no graft rejection and minimal antiglaucoma medications. No complications were noticed in form of phthisis bulbi or graft failure [34].

\section{Conclusion}

Graft survival and permanent irreversible visual loss due to glaucoma are two important factors to be managed in PKG. Avoidance of predisposing factors and aggressive management is advocated to salvage the graft. Newer tools like UBM and ASOCT should be used to diagnose this entity at earliest. Long-term vigilance is required to control IOP as PAS formation is progressive and any intervention can trigger graft rejection and subsequently graft failure. Newer advances like use of the femtosecond laser to cut the host and donor buttons is an attempt to improve wound architecture and limit astigmatism. It allows closure with fewer sutures or tissue glue which decreases angle distortion and chances of PKG. Deep anterior lamellar keratoplasty for anterior corneal diseases has the advantage of maintaining the patient's own endothelium which minimally affects the angle architecture. The newest trend of endothelial keratoplasty (EK) or DSAEK lead to less angle distortion anterior to the TM and crowding of angle as compared to PK. Less incidence of PKG is anticipated with all these new surgical modalities.

\section{References}

1. Foulks GN (1987) Glaucoma associated with penetrating keratoplasty. Ophthalmology 94(7): 871874.

2. Wilson SE, Kaufman HE (1990) Graft failure after penetrating keratoplasty. Surv Ophthalmol 34(5): 325-356.

3. Ayyala RS (2000) Penetrating Keratoplasty and glaucoma. Survey Ophthalmol 45(2): 91-105.

4. Irvine AR, Kaufman HE (1969) Intraocular pressure following penetrating keratoplasty. Am J Ophthalmol 68: 835-844.

5. Goldberg DB, Schanzlin DJ, Brown SI (1981) Incidence of increased intraocular pressure after keratoplasty. Am J Ophthalmol 92(3): 372-377.

6. Kirkness CM, Moshegov C (1988) Post-keratoplasty glaucoma. Eye 2: 19-26.

7. Olson RJ, Kaufman HE (1977) A mathematical description of causative factors and prevention of elevated intraocular pressure after keratoplasty. Invest Ophthalmol Vis Sci 16(12): 1085-1092.

8. Zimmerman TJ, Krupin T, Grodzki W, Waltman SR (1978) The effect of suture depth on outflow facility in penetrating keratoplasty. Arch Ophthalmol 96(3): 505-506.

9. Zimmerman $\mathrm{T}$, Olson $\mathrm{R}$, Waltman $\mathrm{S}$, Kaufman $\mathrm{H}$ (1978) Transplant size and elevated intraocular pressure. Postkeratoplasty. Arch Ophthalmol 96(12): 2231-2233.

10. McMillan F, Forster RK (1975) Comparison of MacKay-Marg, Goldmann, and Perkins tonometers in abnormal corneas. Arch Ophthalmol 93(6): 420-424.

11. Doughty MJ, Zaman ML (2000) Human corneal thickness and its impact on intraocular pressure 
measures: a review and meta-analysis approach. Surv Ophthalmol 44(5): 367-408.

12. Rubinfeld RS, Cohen EJ, Laibson PR, Arentsen JJ, Lugo $\mathrm{M}$, et al.(1998) The accuracy of finger tension for estimating intraocular pressure after penetrating keratoplasty. Ophthalmic Surg Lasers 29(3): 213-215.

13. Dada T, Aggarwal A, Vanathi M, Gadia R, Panda A, et al. (2008) Ultrasound biomicroscopy in opaque grafts with post- penetrating keratoplasty glaucoma. Cornea 27(4): 402-405.

14. Moghimi S, Vahedian Z, Fakhraie G, Ghaffari R (2012) Ocular biometry in the subtypes of angle closure: An anterior segment optical coherence tomography study. American Journal of Ophthalmol 155(4): 664673.

15. Memarzadeh F, Li Y, Francis BA, Smith RE, Gutmark J, et al. (2007) Optical coherence tomography of anterior segment in secondary glaucoma with corneal opacity after penetrating keratoplasty. British Journal of Ophthalmol 91(2): 189-192.

16. Dada T, Shah B M, BaliS J, Bansal N, Panda A, et al. (2011) Anterior segment OCT imaging in opaque graft with secondary glaucoma following tectonic penetrating keratoplasty for perforated corneal ulcer. Eye 25(11): 1522-1524.

17. Karadag O, Kugu S, Erdogan G, Kandemir B, Ozdil SE, et al. (2010) Incidence of and risk factors for increased intraocular pressure after penetrating keratoplasty. Cornea 29(3): 278-282.

18. Reinhard T, Kallmann C, Cepin A, Godehardt E, Sundmacher R (1997) The influence of glaucoma history on graft survival after penetrating keratoplasty. Graefes Arch Clin Exp Ophthalmol 235(9): 553-557.

19. Vajpaye RB, Dada T, Ray M, Tandon R, Sethi A, et al. (2011) Oversized corneal grafts for corneal opacities with iridocorneal adhesions. Ophthalmology 108(11): 2026-2028.

20. Konowal A, Morrison JC, Brown SV, Cooke DL, Maguire LJ, et al. (1999) Irreversible corneal decompensation in patients treated with topical dorzolamide. Am J Ophthalmol 127(4): 403-406.
21. Epstein DL, Grant WM (1977) Carbonic anhydrase inhibitor side effects. Serum chemical analysis. Arch Ophthalmol 95(8): 1378-1382.

22. Perry HD, Donnenfeld ED, Kanellopoulos AJ, Grossman GA (1997) Topical cyclosporin A in the management of postkeratoplasty glaucoma. Cornea 16(3): 284-288.

23. Van Meter WS, Allen RC, Waring GO, Stulting RD (1988) Laser trabeculoplasty for glaucoma in aphakic and pseudophakic eyes after penetrating keratoplasty. Arch Ophthalmol 106(2): 185-188.

24. Skuta GL, Beeson CC, Higginbotham EJ, Lichter PR, Musch DC, et al. (1992) Intraoperative mitomycin versus postoperative 5-fluorouracil in high risk glaucoma filtering surgery. Ophthalmology 99(3): 438-444.

25. Ishiokaa M, Shimazakia J, Fujishimaa $H$, Shimmuraa $S$, Tsubotaa K, et al. (2000)Trabeculectomy with mitomycin $\mathrm{C}$ for post-keratoplasty glaucoma. British Journal of Ophthalmol 84(7): 714-717.

26. Ayyala RS, Pieroth L, Vinals AF, Goldstein MH, Schuman JS, et al. (1998) Comparison of mitomycin C trabeculectomy, glaucoma drainage device implantation, and laser neodymium: YAG cyclophotocoagulation in the management of intractable glaucoma after penetrating keratoplasty. Ophthalmology 105(8): 1550-1556.

27. Raj A, Dhasmana R, Bahadur H (2017) Comparative evaluation of trabeculectomy with releasable suture versus subconjunctival Mitomycin $\mathrm{C}$ in post keratoplasty glaucoma. Sudanese J Ophthalmol 9(1): 16-21.

28. Kirkness CM (1987) Penetrating keratoplasty, glaucoma and silicone drainage tubing. Dev Ophthalmol 14: 161-165.

29. Kirkness CM, Ling Y, Rice NS (1988) The use of silicone drainage tubing to control post-keratoplasty glaucoma. Eye 2(Pt 5): 583-590.

30. Sherwood MB, Smith MF, Driebe Jr WT, Stern GA, Beneke JA, et al. (1993) Drainage tube implants in the treatment of glaucoma following penetrating keratoplasty. Ophthalmic Surg 24(3): 185-189. 
31. Arroyave CP, Scott IU, Fantes FE, Feuer WJ, Murray TG (2001) Corneal graft survival and intraocular pressure control after penetrating keratoplasty and glaucoma drainage device implantation. Ophthalmology 108(11): 1978-1985.

32. Sidoti PA, Mosny AY, Ritterband DC, Seedor JA (2001) Pars plana tube insertion of glaucoma drainage implants and penetrating keratoplasty in patients with coexisting glaucoma and corneal disease. Ophthalmology 108(6): 1050-1058.
33. Ritterband DC, Shapiro D, Trubnik V, Marmor M, Meskin S, et al. (2007) Cornea Glaucoma Implant Study Group (COGIS). Penetrating keratoplasty with pars plana glaucoma drainage devices. Cornea 26(9): 1060-1066.

34. Ocakoglu O, Arslan OS, Kayiran A (2005) Diode laser transscleralc cyclophotocoagulation for the treatment of refractory glaucoma after penetrating keratoplasty. Curr Eye Res 30(7): 569-574. 